\title{
Femmes, genre et colonisations
}

Pascale Barthélémy, Luc Capdevila et Michelle Zancarini-Fournel

\section{Q OpenEdition}

\section{Journals}

\section{Édition électronique}

URL : https://journals.openedition.org/clio/9994

DOI : $10.4000 /$ clio.9994

ISSN : 1777-5299

Éditeur

Belin

Édition imprimée

Date de publication : 1 mai 2011

Pagination : 7-22

ISBN : 978-2-8107-0157-5

ISSN : 1252-7017

Référence électronique

Pascale Barthélémy, Luc Capdevila et Michelle Zancarini-Fournel, «Femmes, genre et colonisations », Clio. Femmes, Genre, Histoire [En ligne], 33 | 2011, mis en ligne le 01 mai 2013, consulté le 26 avril 2022. URL : http://journals.openedition.org/clio/9994; DOI : https://doi.org/10.4000/clio.9994 


\title{
Femmes, genre et colonisations
}

\author{
Pascale BARTHÉLÉMY, \\ Luc CAPDEVILA \& Michelle ZANCARINI-FOURNEL
}

Sobrement intitulé "Colonisations $»^{1}$ ce numéro est le produit d'une envie et d'une actualité historiographique. L'envie de voir et penser large ${ }^{2}$, d'ouvrir davantage la revue à des espaces non occidentaux, de rendre possible une réflexion sur la circulation des modèles et les connexions entre les différents espaces colonisés ${ }^{3}$. Cette envie participe du renouveau de l'histoire coloniale et impériale outre-Atlantique, de son développement en France, et de la faible visibilité des femmes et du genre dans ce champ ${ }^{4}$. Les débats récents sur le passé national ont aussi joué un rôle dans le choix de cette thématique, tant ils ont à la fois clivé et stimulé le champ des études coloniales ${ }^{5}$.

1 Le titre envisagé dans un premier temps « le genre de la colonisation » a semblé superfétatoire pour une revue toute entière consacrée à l'histoire des femmes et du genre.

2 Douki \& Minard 2007.

3 Etemad 2000.

4 Saada 2001 ; Burbank \& Cooper 2010 ; Douki \& Minard 2007 ; Annales. HSS 2008. CLIO. Histoire, Femmes et Sociétés a célébré ses quinze ans d'existence le 28 janvier 2011 autour du thème «Femmes, genre et histoire globale ». L'ensemble de la journée est disponible en ligne : http://www.archivesaudiovisuelles.fr/FR/

5 Bertrand 2006b et Coquery-Vidrovitch 2009. Le dynamisme de ce champ se manifeste notamment par la multiplication des séminaires ces trois dernières années : «Empires. Histoire des colonisations » à l'ENS Ulm; «Histoire des colonisations européennes. XIX $-\mathrm{XX}^{\mathrm{e}}$ siècles » au Centre d'histoire de Sciences Po ; «Histoire transnationale des pensées raciales, XVIII $-\mathrm{XX}^{\mathrm{e}}$ » à New York University - Paris, ainsi que par les inscriptions en thèse sur ces questions. 


\section{Itinéraire d'une rencontre}

Malgré la multiplication des travaux et l'ouverture de plus en plus nette à une histoire sociale et culturelle, les historiens du colonial comme les spécialistes des sociétés d'Afrique, d'Asie ou des Amériques sont encore peu nombreux à faire une place aux femmes et au genre. Les raisons anciennes de cette occultation ont été maintes fois soulignées ${ }^{6}$. Rappelons simplement qu'au lendemain des indépendances, l'analyse critique du fait colonial et les nécessités des constructions nationales ont suscité un processus de «fabrique des héros $»^{7}$ excluant, de fait, les femmes. Dans les années 1980, le mouvement des Subaltern Studies lancé simultanément en Inde et en Grande-Bretagne, malgré son souci de restituer le rôle et la parole des populations jusque-là marginalisées, s'est d'abord peu intéressé aux femmes. Inspirés par les Cahiers de prison de Gramsci ${ }^{8}$, des historiens indiens et leur chef de file, Ranajit Guha, ont proposé une autre lecture des archives coloniales britanniques, qui remettait en cause l'historiographie nationaliste antérieure, centrée sur le rôle des élites indiennes et sur la mobilisation des masses "par le haut». Ces travaux, publiés dans la revue Subaltern Studies dont le premier numéro est paru en 1982, ont suscité depuis de nombreuses critiques dont celle d'avoir essentialisé la « conscience subalterne » et donc, aussi, de ne pas avoir intégré la variable du genre. Quant aux études postcoloniales, qui ont émergé à la même époque d'abord dans le champ littéraire, elles interrogent les rapports de domination au prisme du genre, mais il leur a été reproché de céder à la seule étude des discours et de proposer des analyses très théoriques ${ }^{9}$. C'est cependant les chercheurs anglophones qui, les premiers, ont ajouté la

6 Voir Hugon 2004.

7 L'expression est empruntée à Centlivres, Fabre \& Zonabend 1998, qui cependant n'abordent pas les espaces non européens.

8 Gramsci 1992 [1934] : 309-317.

9 Pour des synthèses en français sur les Subaltern Studies et les études postcoloniales voir Diouf 1999; Pouchepadass 2000; Smouts 2007. Lire également l'article fondateur de Gayatri Chakravorty Spivak, désormais traduit en français : 2009 [1988]. Pour un ensemble d'articles récents voir Berger \& Varikas 2011. Pour une approche critique Ludden 2002. 
variable « sexe » à celle de « classe » et de « race $»^{10}$. Ces recherches concernent prioritairement les anciennes colonies britanniques, même si des historiennes se spécialisent sur le Maghreb11. L'état des lieux historiographique coordonné par Jean Allman et Antoinette Burton pour le Journal of Colonialism and Colonial History en 2003 témoigne de cette vitalité 12 .

En France, jusqu'aux années 1980-1990, les spécialistes d'histoire des femmes ont peu abordé le champ colonial ou les sociétés non occidentales d'une façon générale. Pionnières, Yvonne Knibiehler et Régine Goutalier lancèrent à Aix-en-Provence un programme de recherche sur «Femmes et colonisation $»^{13}$. Leur entreprise, centrée sur le Maghreb, est restée isolée. C'est à partir du début des années 1990 qu'un certain nombre d'initiatives permettent l'accumulation des connaissances en français et sur les colonies françaises comme l'acclimatation des recherches en langue anglaise. Signe d'un champ désormais bien constitué, deux séminaires ont été créés récemment qui croisent le genre et la colonisation ${ }^{14}$.

Par ailleurs, et de plus en plus, des chapitres, des paragraphes consacrés aux femmes, pénètrent dans les études générales. C'est le cas, par exemple, dans la thèse de Laurence Monnais-Rousselot qui, au fil d'une histoire de la médecine en Indochine française, aborde le rôle des sages-femmes et de la médicalisation de la maternité15. Cette intégration de l'histoire des femmes et du genre à l'histoire générale $\mathrm{du}$ fait colonial est visible dans deux synthèses publiées au début des années 2000, Le Livre noir du colonialisme et Colonisation: droit d'inventaire ${ }^{16}$. Dans la première, Arlette Gautier rappelle que « c'est la

10 Voir, par exemple, McClintock 1995 ; Cooper \& Stoler 1997 ; Clancy-Smith \& Gouda 1998 ; Roach Pierson \& Chaudhuri 1998; Stoler 2002; Levine 2004 ; Fischer-Tiné \& Gehrmann 2009.

11 Clancy-Smith 1996, 2000 ; Clancy-Smith \& Gouda 1998.

12 Allman \& Burton 2003.

13 Knibiehler \& Goutalier 1985.

14 Séminaire de Françoise Gaspard et Christelle Taraud, à New York UniversityParis, Le genre en situation coloniale et post-coloniale; séminaire de Brigitte Rollet à l'Institut de Londres à Paris, Genre et cultures dans un contexte colonial et post-colonial.

15 Monnais-Rousselot 1999.

16 Gautier 2003 : 569-607 et Liauzu 2004. 
construction même des genres, c'est-à-dire à la fois ce qui était attendu en fonction du sexe et les rapports entre les sexes, qui a été bouleversée par les différentes colonisations $»^{17}$. Mais, notons l'absence de référence aux femmes (sinon ponctuellement dans certains chapitres) dans le Dictionnaire de la France coloniale dirigé par Jean-Pierre Rioux chez Flammarion en 2007. C'est donc du côté des revues spécialisées dans le champ que les choses bougent d'abord. En 2008, la revue Sextant. Revue du groupe interdisciplinaire sur les femmes et le genre en Belgique a consacré un numéro aux "colonialismes $»^{18}$. Au sein de Clio HFS, les "colonisations » ont aussi une histoire. Depuis sa création, la revue a publié trois dossiers spécifiques - «Femmes d'Afrique » en 1997, «Femmes du Maghreb » en 1999 et « Amériques métisses » en 2008.

En 1997, pour la première fois, une historienne extérieure au comité de rédaction, Catherine Coquery-Vidrovitch était invitée à partager son savoir sur l'Afrique subsaharienne. Soucieux de se démarquer de l'anthropologie, le numéro «Femmes d'Afrique » arpente les XIXe et XX $\mathrm{XX}^{\mathrm{e}}$ siècles et examine la période coloniale, mais aussi postcoloniale, à travers la question du genre des nouveaux États indépendants et des cinémas africains. En 1999, «Femmes du Maghreb» dirigé par Agnès Fine et Claudine Leduc, replace la colonisation dans l'histoire longue des pays considérés, depuis l'occupation romaine jusqu'aux indépendances. Il intègre l'histoire des féminismes et des nationalismes comme celle des représentations littéraires dans les contes et les romans. Les articles et comptes rendus qui ont abordé la colonisation dans d'autres numéros portent quasiment tous sur le Maghreb et concernent l'éducation ${ }^{19}$, le corps et les sexualités. Dans le numéro «ProstituéEs» Christelle Taraud a étudié le cas des filles soumises « indigènes ${ }^{20}$ du quartier réservé de Casablanca dans les années 1920-1950. C’est aussi à l'initiative de Clio

17 Gautier $2003: 570$.

18 Voir le compte rendu dans ce numéro.

19 Clancy-Smith 1996, 2000 ; Clancy-Smith \& Gouda 1998.

20 Sur l'emploi de ce terme avec ou sans guillemets dans les articles de ce numéro, le choix des auteur-e-s a été respecté. L'absence de guillemets renvoie à l'étymologie du terme qui désigne - sans connotation péjorative - les populations locales non européennes. Voir Taraud 2003. 
HFS qu'ont été organisés à Toulouse en 2002 dans le cadre du troisième colloque international de la recherche féministe francophone une table ronde et des ateliers sur "Colonisation, décolonisation, post-colonialisme et genre ». Un livre, dirigé par Anne Hugon, est issu de ces rencontres ${ }^{21}$.

Depuis ses débuts, Clio HFS a fait des choix en fonction de plusieurs impératifs : la nécessité de ne pas tout réduire au moment colonial22 $^{22}$ de ne pas se couper d'autres disciplines (anthropologie, ethnologie, sociologie, droit); d'utiliser des sources orales et écrites (et aussi la littérature, le cinéma, la photographie); de s'intéresser aux questions de virilisation ou/et de féminisation des individus dans les projets coloniaux. Ainsi, l'analyse par Catherine Marand-Fouquet des statues des escaliers de la gare Saint-Charles à Marseille montrait une représentation féminisée des différentes colonies ${ }^{23}$. Signalons enfin en Varia du volume "Amériques métisses", l'article novateur d'Emmanuel Blanchard sur l'affrontement de masculinités divergentes entre les immigrants algériens, célibataires d'occasion, et la police parisienne, marquée par une homosociabilité masculine.

Si Clio HFS fut pionnière, la rencontre entre histoire des femmes et du genre et histoire de la colonisation se produit timidement dans les revues généralistes : les femmes sont absentes du numéro de Genèses en 2007 «La parole est aux indigènes » comme de celui des Annales. Histoire, Sciences sociales intitulé «Empires » en 2008. Signalons toutefois que Genèses a intégré un article sur l'Afrique dans son numéro «Femmes, genre et histoire » dès 1991 et que les Annales ont publié un article sur les écritures féminines dans "Cultures écrites en Afrique » en 200924. Cependant, et malgré ces avancées, l'approche reste considérée par nombre d'historien-ne-s comme une façon périphérique d'aborder le fait colonial, consistant à apporter des connaissances supplémentaires mais non décisives. Ce numéro espère

21 Hugon 2004.

22 Bertrand 2006a. L'expression entend souligner que «l'historicité politique moderne des sociétés d'Asie, d'Océanie ou d'Afrique ne saurait se résumer à leur mise sous séquestre provisoire par les États européens ».

23 Photographies commentées dans CLIO HFS 2000 : 188-191.

24 Goerg 1991 : 144-160 ; Barthélémy 2009 : 825-852. 
convaincre du contraire. Dans une perspective d'histoire sociale, les articles interrogent la manière dont la colonisation a fabriqué des colonisateurs comme des colonisé-e-s, dont pourtant l'existence ne se réduisait pas à être, seulement, l'un ou l'autre ${ }^{25}$.

\section{Repenser les catégories, interroger les pratiques}

Les Indes néerlandaises, l'Inde britannique, le Soudan français, le Congo belge, la Guinée, l'Indochine, la Syrie, l'Argentine du dernier XIX ${ }^{e}$ siècle, l'Égypte hellénistique et le Maghreb sont représentés dans un numéro qui éclaire ainsi d'une certaine façon la colonisation comme processus global, affectant tous les continents sur plusieurs siècles. Il ne s'agit pas seulement de permettre une histoire comparée des Empires, mais de suggérer l'existence de circulations entre les différentes parties du monde. Les articles permettent aussi de lier des histoires particulières à des analyses plus larges d'un système de domination. En utilisant des sources originales ce numéro confirme, s'il en était besoin, que les chercheurs n'ont pas à souffrir de l'absence d'archives. Bien plus, les sources politiques, judiciaires, administratives, les archives privées (ainsi le journal de dédicaces d'une collégienne vietnamienne présenté par Phuong Bui Tran), les sources orales donnent accès aux pratiques, et pas seulement aux représentations. Elles permettent d'identifier des stratégies d'opposition, d'émancipation, d'adaptations et/ou de détournements par les colonisé-e-s des normes imposées par les autorités coloniales. En s'interrogeant sur le sexe et le genre, les articles retravaillent la question des hiérarchies en «situation coloniale ${ }^{26}$. Ils montrent des conjonctions d'intérêts possibles entre hommes européens et congolais ou syriens, des relations complexes entre hommes blancs et femmes africaines ou indonésiennes, des hiérarchies de classe au sein du groupe des femmes colonisées, et des affinités, mais aussi des incompréhensions entre Européennes et femmes indigènes. Ces configurations sont abordées au travers de deux grandes thématiques

\footnotetext{
25 A. Memmi insistait déjà dans les années 1950 sur ce processus de construction des catégories mais tendait à réduire l'existence des Européens et des «Autres » au seul statut de colonisateur ou de colonisé. Memmi 1985 [1957].

26 Balandier 1951.
} 
- celle de l'intime et celle du politique - qui se croisent et se recoupent.

Frances Gouda, Marie Rodet et Amandine Lauro traitent sous différents angles la question des relations intimes entre colonisateurs et colonisé-e-s mais aussi au sein des sociétés locales. Frances Gouda analyse sur la longue durée la construction des catégories à travers la question des métis dans les Indes néerlandaises qu'elle compare à l'Inde britannique et à l'Indochine française. Elle complète ainsi le numéro «Amériques métisses » dont le dossier permettait d'observer l'affirmation des identités masculines et féminines dans les relations interculturelles, dans la soumission individuelle et collective à un groupe ethnique dominant, dans la conscience d'appartenir à une casta (caste). C'est aussi la question des hiérarchies entre femmes qu'aborde l'article d'Anne-Emmanuelle Veïsse. À travers une étude fine de la documentation papyrologique, elle va à l'encontre d'une historiographie qui insiste sur la supériorité de statut des femmes égyptiennes (colonisées) sur celui des femmes grecques, colonisatrices mais contraintes d'avoir recours à un kurios (tuteur légal). Elle montre ainsi que l'incapacité juridique des femmes grecques fut relative.

Marie Rodet examine, au travers de quatre affaires traitées par l'administration coloniale entre 1902 et 1906, les relations intimes qui ont pu se nouer au Soudan français (actuel Mali) au tournant du XIX et du XXe siècle, entre des femmes esclaves et leurs maitres ou leurs maitresses européen-ne-s ou africains ${ }^{27}$. À partir des archives des cercles, circonscriptions administratives de base, elle souligne que les abus sexuels sur les femmes ont largement dépassé l'étape de la conquête militaire, mais identifie aussi des stratégies d'émancipation de la part de certaines femmes dont elle retrace les parcours complexes. À la charnière d'une triple historiographie - celle du genre, de la justice coloniale et des cultures de l'écrit en Afrique ${ }^{28}$, Amandine Lauro décortique un corpus de 200 lettres envoyées par des hommes congolais à l'administration coloniale belge pour se plaindre des femmes de leur famille entre les années 1910 et 1950.

27 Sur le croisement entre histoire de la justice et du genre en situation coloniale voir Coquery-Vidrovitch 2007.

28 Voir Ficquet \& M'Bodj-Pouye 2009. 
Elle analyse en terme de crise du mariage - mais aussi d'une reconfiguration des masculinités - cette profusion d'écrits.

Les articles de Frances Gouda, de Marie Rodet et d'Amandine Lauro dessinent une chronologie de la manière dont l'administration coloniale a envisagé les relations intimes entre Européens et femmes indigènes et suggèrent de creuser la circulation des modèles entre les différents Empires en matière de «moralisation» des sociétés colonisées $^{29}$. L'intime est enfin central dans le riche bilan historiographique réalisé par Christelle Taraud sur le Maghreb colonial. Elle rend compte de la richesse - et du caractère récent des travaux sur les Européennes, souligne la concentration des recherches sur l'Algérie et identifie de nombreuses zones d'ombre, notamment sur l'articulation entre femmes, genre et politique, une thématique abordée ici par Nupur Chaudhuri, Elizabeth Thompson et Phuong Bui Tran.

La première examine le rôle des Indiennes, entre féminisme et nationalisme, de la fin du XIXe à l'indépendance en 1947. À partir de sources britanniques classiques mais aussi de documents inédits en bengali, elle identifie les différentes phases et formes de l'engagement des femmes dans la lutte contre le pouvoir colonial. Ainsi apparaissent des connexions entre le féminisme britannique (celui, par exemple, d'Annie Besant) et les mouvements féminins en Inde. Cet article fait écho à celui d'Elizabeth Thompson, sur les mouvements féminins dans le cadre du mandat français en Syrie entre les années 1920 et 1946. Elle montre en effet que les Syriennes, militant en faveur de réformes sociales et politiques, s'inspirent directement des méthodes de Gandhi en appelant au boycott des produits étrangers et au développement de l'artisanat local, notamment féminin. E. Thompson suggère de nombreuses pistes pour étudier les circulations internationales des luttes. Elle met aussi l'accent sur le rôle de l'instruction des filles dans la mobilisation politique. Une thématique que l'on retrouve dans le parcours scolaire de Kadidiatou Diallo Telli, une des premières institutrices de Guinée

29 Voir Cooper \& Stoler 1997. Amandine Lauro a montré qu'une circulaire britannique de 1909 sur le concubinage a inspiré la législation belge au Congo. Lauro 2005. 
française, et qui est également abordée par Phuong Bui Tran dans sa présentation d'un journal de collégienne vietnamienne dans les années 1930 et 1940. Ces témoignages rendent vivant le ressenti des jeunes filles scolarisées en internat en Afrique occidentale française comme en Indochine. Phuong Bui Tran souligne aussi que cette adhésion au modèle d'éducation occidental cohabite avec une conscience politique et un engagement ultérieur dans les guerres d'indépendance.

L'article d'E. Thompson montre enfin la complexité des liens entre genre et nationalisme. Alors que les femmes adressent des revendications sociales tout à fait neuves à l'autorité mandataire en Syrie, elles se retrouvent marginalisées lorsqu'une législation entérine des droits, à la fois par l'alliance entre les Français et les élites conservatrices, et par celle qui fait se rejoindre islamistes et nationalistes masculins. E. Thompson interroge aussi la «situation coloniale » puisque, dans le cas du mandat français en Syrie, ce n'est pas tout à fait de cela qu'il s'agit. Cette question est prégnante dans les travaux sur l'Amérique latine, et l'article d'Ana Teruel s'en fait l'écho.

\section{La question du colonialisme interne}

En effet, le processus de colonisation n'a pas cessé en Amérique à la suite du cycle des indépendances entre la fin du XVIII ${ }^{e}$ et le début du XIX ${ }^{e}$ siècle. Certes, les jeunes républiques dénoncèrent l'asymétrie des relations que les monarchies européennes leur avaient imposée ; et en prônant la suprématie des droits individuels sur les droits collectifs elles engagèrent conjointement la démolition de l'édifice d'Ancien Régime sur lequel reposait l'ordre colonial impérial. Mais, simultanément, les nouveaux États inventèrent des formes de discriminations raciale et ethnique qui élevaient des barrières - pour certaines invisibles - à l'intérieur de la société ; et elles poursuivirent le programme de conquête des immenses territoires indiens encore libres de toute présence occidentale au début du XIX ${ }^{e}$ siècle. C'est dans les années 1950-1960 que la sociologie mexicaine a forgé le concept de "colonialisme interne $»^{30}$ afin de mettre en évidence la situation coloniale pérennisée dans l'asymétrie des relations

30 Gonzalez Casanova 1964. 
économiques, sociales, politiques et culturelles entre les communautés paysannes amérindiennes et les sociétés nationales. Par la suite, notamment depuis que les sociétés latino-américaines se sont orientées vers la reconnaissance de leurs caractères multiethnique et pluriculturel dans les années $1980^{31}$, les historiographies se sont profondément renouvelées en interrogeant l'histoire de leur interculturalité, ainsi que les formes républicaines de la colonisation.

Cette histoire est complexe. Il semble qu'au début du XIXe siècle les élites républicaines d'Amérique latine envisagèrent de bâtir un État national multiculturel ${ }^{32}$. Puis, dans la seconde moitié du XIX ${ }^{e}$ siècle, inspirées par les modèles européens, elles convergèrent radicalement pour construire des États-nationaux unitaires en imposant leur autorité sur des territoires qu'elles considéraient comme partie intégrante de l'espace national. C'est ce processus qu'Ana Teruel étudie dans le nord de l'Argentine à la fin du XIX e et au début du XX siècle. La disciplinarisation ${ }^{33}$ des Indiens par les missionnaires, les exploitants, les militaires consistait à les faire sortir d'un état censé être de barbarie. Il s'agissait de les sédentariser, de les vêtir, de les mettre au travail pour les élever d'abord à la condition d'homme et de femme selon les canons des sociétés nationales, avant d'en faire des chrétiens, puis à terme, peut-être, des citoyens. Cette expansion coloniale tardive, en transformant l'environnement, le mode de vie, l'organisation sociale, a constitué, jusqu'au $\mathrm{XX}^{\mathrm{e}}$ siècle, un lieu majeur de mutation du genre des populations dites désormais "indigènes ». L'une des conséquences de l'action missionnaire sur les groupes de collecteurs matricentrés fut de renforcer la domination masculine en désignant les hommes comme les interlocuteurs salariés privilégiés des colons. Les hiérarchies masculines furent ainsi elles aussi profondément altérées. En s'étendant sur ces territoires et en mettant un terme aux guerres entre ethnies, les États de facto ont effacé les deux figures extrêmes de la virilité à partir desquelles se déclinaient les masculinités indiennes correspondant à ces espaces nouvellement

\footnotetext{
31 Bertrand \& De Roux 2008.

32 Pinto Rodriguez 2003.

33 Dans le sens que Michel Foucault donne à «la discipline "fabrique" des individus ». Foucault 1975 : 172.
} 
conquis : celle du guerrier et celle du captif. Mais ils firent émerger de nouvelles incarnations consolidant la domination masculine à travers la figure actualisée du «cacique », celle du contremaître, celle du garde ou du soldat, voire celle du maître d'école.

\section{Les masculinités à l'épreuve de la colonisation}

L'ambition de ce numéro est aussi de montrer que l'expansion coloniale commence à être étudiée comme un moment central de redéfinition des masculinités. En effet, tant le caractère viril des colonisateurs incarné par des figures dominatrices d'hommes blancs - l'officier, le missionnaire, l'administrateur, l'ingénieur, le planteur -, que la rhétorique de dévirilisation des peuples soumis, réduits à une condition subalterne, furent exacerbés ${ }^{34}$. Mais, au-delà de l'analyse des discours et des représentations produits dans l'événement, les études empiriques sur le devenir des masculinités dans les territoires sous domination française demeurent aujourd'hui peu nombreuses, alors que l'on dispose déjà d'une historiographie conséquente sur les colonies anglophones ${ }^{35}$. Or, l'impact de la colonisation sur les hommes fut aussi complexe que sur les femmes. Si la conquête puis la domination des populations en altérant les hiérarchies masculines soumises a affecté leur éclat et leur autorité, elles s'accompagnent conjointement de circulations culturelles et de transferts de pouvoirs qui favorisent des ajustements et des configurations nouvelles dans les rapports de genre.

L'article de Vincent Joly interroge dans ce volume les conditions historiques et les ressorts culturels qui ont participé à l'émergence de la notion de « race guerrière » dans les Indes sous domination anglaise comme en Afrique occidentale française. Il montre en quoi la colonisation produit des virilités subalternes mais valorise aussi des groupes humains selon les canons de la virilité militaire (résistance, endurance, force, agressivité...). Le procédé pourrait sembler antinomique avec le projet colonial, si ce n'est que le propre de celuici a été d'instituer des hiérarchies fondées sur les discriminations

34 Sinha 1995.

35 Pour l'Afrique voir Lindsay \& Miescher 2003 et sur l'homosexualité dans les Empires Aldrich 2003. 
entre les « races » et de les hiérarchiser. L'idée que des ethnies étaient plus guerrières que d'autres traverse les Empires français et anglais ; un phénomène culturel et militaire également observable en Amérique au $\mathrm{XX}^{\mathrm{e}}$ siècle pour certains groupes amérindiens. Mais, ce que montre Vincent Joly, c'est aussi un phénomène particulier de réappropriation par certaines ethnies d'une identité guerrière réinventée ou réactivée par les colonisateurs.

Amandine Lauro propose une autre lecture des transformations des masculinités dans le Congo belge des années 1930 aux années 1950. Ainsi, les autorités administratives en particulier, en recherchant des interlocuteurs et des partenaires, ont consolidé les hiérarchies masculines à travers la "régénération » des tribunaux indigènes coutumiers confiés généralement à des chefs et qui s'occupaient des affaires de droit civil entre indigènes. Ils existaient dans la plupart des systèmes coloniaux, en Amérique y compris. Ce que souligne ici Amandine Lauro c'est moins une crise de la masculinité liée aux départs des femmes du domicile conjugal, qu'une appropriation des codes et normes occidentaux en matière conjugale que les Congolais réutilisent pour se faire entendre de l'administration. La confrontation des modèles de genre est au cœur de ces affaires de justice. Désarroi des hommes et des femmes confrontés à l'évolution de la société induite par la colonisation, appropriation des normes de genre occidentales, inertie des pratiques dans les rapports hommes/femmes et les représentations des cultures indigènes, attitudes des administrateurs européens enclins à soutenir l'autorité des maris, forment le système complexe de ce qui correspond à une recomposition du genre au cours du premier XXe siècle.

Il est donc question de «colonisations » diverses dans ce numéro qui veut à la fois aborder des situations de référence, apporter des éclairages sur des espaces plus singuliers, poser la question du «colonial», introduire une histoire des masculinités. Tout en proposant une histoire sociale attentive à montrer ce que l'approche par les femmes et le genre permet en termes de compréhension de ce que fut l'expérience historique de la colonisation, il s'agit aussi d'ouvrir des pistes pour des recherches ultérieures plus attentives encore aux circulations et aux connexions à l'échelle impériale. 


\section{Bibliographie}

ALDRICH Robert, 2003, Colonialism and Homosexuality, Londres, Routledge.

Allman Jean \& Antoinette BurTon, 2003, «Destination Globalization? Women, Gender and Comparative colonial histories in the New Millenium », Journal of Colonialism and Colonial History, 4/1.

AUSLANDER Leora \& Michelle ZANCARINI-FOURNEL (dir.), 2000, «Le genre de la nation », CLIO, Histoire, Femmes et Societés.

BALANDIER Georges, 1951, "La situation coloniale : approche théorique », Cahiers internationaux de sociologie, 11 , p. 44-79.

BARTHÉLÉMY Pascale, 2009, «Je suis une Africaine... j’ai vingt ans. Écrits féminins et modernité en Afrique occidentale française (c. 1940 - c. 1950) », Annales. Histoire, Sciences sociales, 4, p. 825-852.

—, 2010, Africaines et diplômées à l'époque coloniale (1918-1957), Rennes, Presses Universitaires de Rennes.

Berger Anne \& Eleni VArikas (dir.), 2011, Genre et postcolonialismes. Dialogues transcontinentaux, Paris, Archives contemporaines.

BERTRAND Romain, 2006a, "Les sciences sociales et le moment colonial : de la problématique de la domination coloniale à celle de l'hégémonie impériale », Questions de recherche, 18 (disponible en ligne).

—, 2006b, Mémoires d'Empire : la controverse autour du fait colonial, Bellecombe-en-Baufes, Éditions du Croquant.

Bertrand Michel \& Rodolfo De Roux (dir.), 2008, De l'un au multiple. Dynamismes identitaires en Amérique latine, Toulouse, Presses universitaires du Mirail.

Burbank Jane \& Frederick CoOper, 2008, « Empire, droits et citoyenneté », Annales. Histoire, Sciences sociales, 3, p. 495-531.

-, 2010, Empires in World History: Powers and the Politics of Difference, Princeton, Princeton University Press.

Centlivres Pierre, FABre Daniel \& Françoise ZonABend (dir.), 1998, La fabrique des héros, Mission du Patrimoine ethnologique/Éditions de la MSH.

Chaudhuri Nupur \& Margaret STROBEL (eds), 1992, Western Women and Imperialism: Complicity and Resistance, Bloomington/Indianapolis, Indiana University Press.

Clancy-Smith Julia, 1996, "The Colonial Gaze: Sex and Gender in the Discourses of French North Africa », in Carl L. Brown \& Mathew S. Gordon (eds), FrancoArab Encounters, Beirut, American University of Beirut and Syracuse, New York, Syracuse University Press, p. 201-228. 
—, 2000, «L'école de la rue du Pacha à Tunis : l'éducation de la femme arabe et la “plus grande France” 1900-1914 », CLIO. Histoire, Femmes et Sociétés, 12, p. 33-55.

ClanCY-Smith Julia \& Frances Gouda (eds), 1998, Domesticating the Empire: race, gender and family life in French and Dutch colonialism, Charlottesville, University Press of Virginia.

COOPER Frederick, 2010, Le colonialisme en question. Théorie, connaissance, histoire, Paris, Payot.

COOPER Frederick \& Ann STOLER (eds), 1997, Tensions of Empire: Colonial Cultures in a Bourgeois World, Berkeley, University of California Press.

Coquery-Vidrovitch Catherine, 1994, Les Africaines. Histoire des femmes d'Afrique noire du XIXe au XX siècle, Paris, Éditions Desjonquères.

—, 2009, Enjeux politiques de l'bistoire coloniale, Paris, Agone.

Coquery-Vidrovitch Catherine (dir.), 2007, "Les femmes, le droit et la justice », Cabiers d'Études Africaines, 87-188, XLVII (3-4).

Coquery-Vidrovitch Catherine \& Françoise ThéBaud (dir.), 1997, «Femmes d'Afrique », CLIO. Histoire, Femmes et Sociétés, 6.

Diouf Mamadou, 1999, L’historiographie indienne en débat. Colonialisme, nationalisme et sociétés postcoloniales, Paris, Karthala-Sephis.

DOUKI Caroline \& Philippe MINARD, 2007, « Histoire globale, histoire connectée : un changement d'échelle historiographique ? ", RHMC, 54-4 bis.

«Empires », 2008, Annales. Histoire, Sciences sociales, 63e année, n³.

ETEMAD Bouda, 2000, La possession du monde. Poids et mesures de la colonisation, Bruxelles, Complexe.

FERRO Marc, 1994, Histoire des colonisations, des conquêtes aux indépendances, XIII -XX $^{e}$ siècles, Paris, Seuil.

FERRO Marc (dir.), 2003, Le Livre noir du colonialisme, XVte-XXIe siècle : de l'extermination à la repentance, Paris, Robert Laffont.

FINE Agnès \& Claudine LeduC (dir.), 1999, «Femmes du Maghreb », CLIO. Histoire, Femmes et Sociétés, 9.

FICQUET Eloi \& Aïssatou M'BODj-POuYE (dir.), 2009, «Cultures écrites en Afrique ", Annales. Histoire, Sciences sociales, 64e année, $\mathrm{n}^{\circ} 4$.

Fischer-Tiné Harald \& Susanne GEHRMAnN (eds), 2009, Empires and Boundaries. Rethinking Race, Class and Gender in Colonial Settings, New York, Routledge.

FouCault Michel, 1975, Surveiller et punir. Naissance de la prison, Paris, Gallimard.

Gautier Arlette, 2003, «Femmes et colonialisme », in Marc Ferro (dir.), Le Livre noir du colonialisme, Paris, Robert Laffont, p. 569-607. 
Goerg Odile, 1991, «L'historiographie de l'Afrique de l'Ouest: tendances actuelles », Genèses, « Femmes, genre et histoire », 6, p. 144-160.

Goerg Odile (dir.), 2007, Perspectives bistoriques sur le genre en Afrique, Paris, L'Harmattan.

Gramsci Antonio, 1992 [1934], «Aux marges de l'histoire. Histoire des groupes sociaux subalternes ", in Robert PARIS (dir), Cabiers de prison, 25, Paris, Gallimard, p. 309-317.

GonzÁlez Casanova Pablo, 1964, «Société plurale - colonialisme interne et développement », Tiers-Monde, 18, p. 291-295.

Hugon Anne (dir.), 2004, Histoire des femmes en situation coloniale, Afrique et Asie, XXe siècle, Paris, Karthala.

Knibiehler Yvonne \& Régine Goutalier, 1985, La femme au temps des colonies, Paris, Stock.

Lauro Amandine, 2005, Coloniaux, ménagères et prostituées au Congo belge, 1885-1930, Loverval, Labor.

LAURO Amandine (dir.), 2008, "Colonialismes », Sextant. Revue interdisciplinaire sur les femmes et le genre.

Levine Philippe (ed.), 2004, Gender and Empire, New York, Oxford University Press.

Liauzu Claude (dir.), 2004, Colonisation : droit d'inventaire, Paris, Colin.

Lindsay Lisa L. \& Stephan F. Miescher, 2003, Men and Masculinities in Modern Africa, Portsmouth, Heinemann.

LOCOH Thérèse (dir.), 2008, Genre et sociétés en Afrique, Paris, INED.

LudDen David (ed.), 2002, Reading Subaltern Studies: Critical History, Contested Meaning and The Globalization of South Asia, Londres, Anthem Press.

McClintock Anne, 1995, Imperial Leather: Race, Gender and Sexuality in the Colonial Contest, New York, Routledge.

Memmi Albert, 1985 [1957], Portrait du colonisé. Portrait du colonisateur, Paris, Gallimard.

Midgley Clare, 1998, Gender and Imperialism, Manchester, Manchester University Press.

Monnais-Rousselot Laurence, 1999, Médecine et colonisation, l'aventure indochinoise, 1860-1939, Paris, CNRS éditions.

PINTO RoDríGuEZ Jorge, 2003, La formación del Estado y la nación y el pueblo mapuche. De la inclusión a la exclusión, Santiago de Chile, DIBAM.

POUCHEPADASS Jacques, 2000, « Les Subaltern Studies ou la critique postcoloniale de la modernité », L'Homme, 156, p. 161-186. 
Rodet Marie, 2009, Les migrantes ignorées du Haut-Sénégal (1900-1960), Paris, Karthala.

Roach Pierson Ruth \& Nupur Chaudhuri, 1998, Nation, Empire, Colony: Historicing gender and race, Bloomington, Indiana University Press.

SAADA Emmanuelle, 2001, "La situation coloniale vue d'ailleurs : regards croisés transatlantiques », Cahiers internationaux de sociologie, 110, p. 31-52.

SinHA Mrinalini, 1995, Colonial Masculinity. The "Manly Englishman" and the "Effeminate Bengali" in the late Nineteenth Century, Manchester, Manchester University Press.

SMOUTS Marie-Claude (dir.), 2007, La situation postcoloniale : les postcolonial studies dans le débat français, Paris, Presses de Sciences Po.

SPIVAK Gayatri Chakravorty, 2009 [1988], Les subalternes peuvent-elles parler? Paris, Amsterdam.

STOLER Ann L., 2002, Carnal knowledge and Imperial power: Race and the intimate in colonial rule, Berkeley, University of California Press.

Taraud Christelle, 2003, La prostitution coloniale. Maroc, Algérie, Tunisie, Paris, Payot. 